This item was submitted to Loughborough's Research Repository by the author.

Items in Figshare are protected by copyright, with all rights reserved, unless otherwise indicated.

\title{
Underdetermined blind source separation of temporomandibular joint sounds
}

PLEASE CITE THE PUBLISHED VERSION

PUBLISHER

(C) IEEE

VERSION

VoR (Version of Record)

LICENCE

CC BY-NC-ND 4.0

REPOSITORY RECORD

Took, Clive Cheong, Saeid Sanei, Jonathon Chambers, and Stephen Dunne. 2019. "Underdetermined Blind Source Separation of Temporomandibular Joint Sounds”. figshare. https://hdl.handle.net/2134/5535. 
This item was submitted to Loughborough's Institutional Repository (https://dspace.lboro.ac.uk/) by the author and is made available under the following Creative Commons Licence conditions.

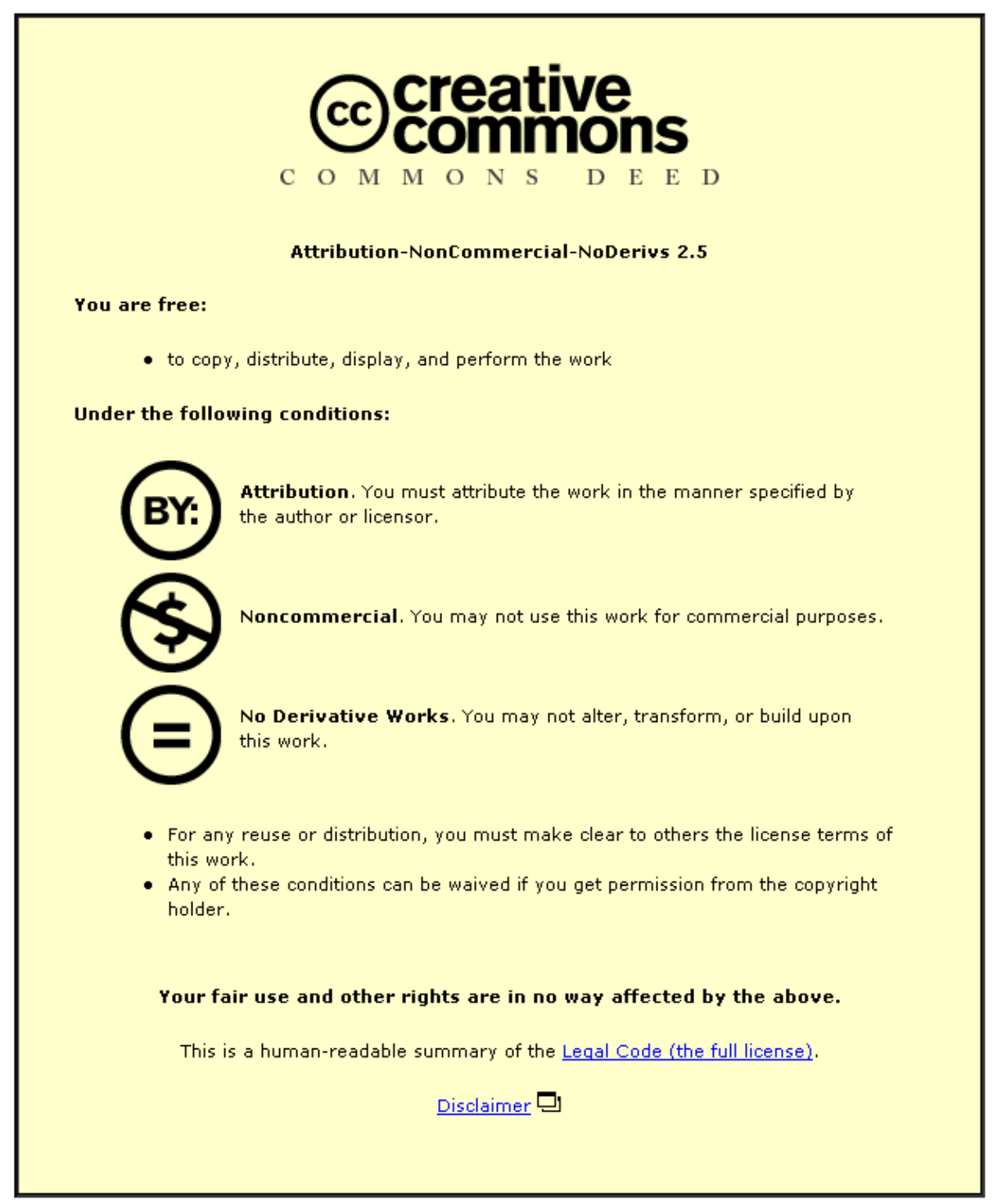

For the full text of this licence, please go to: http://creativecommons.org/licenses/by-nc-nd/2.5/ 
[6] R. Bartenschlager, L. Ahlborn-Laake, J. Mous, and H. Jacobsen, "Nonstructural protein 3 of the hepatitis $\mathrm{C}$ virus encodes a serine-type proteinase required for cleavage at the NS3/4 and NS4/5 junctions," $J$. Virol., vol. 67, pp. 3835-3844, 1993.

[7] M. Eckard, M. Selby, F. Masiarz, C. Lee, K. Berger, K. Crawford, C. Kuo, G. Kuo, M. Houghton, and Q. Choo, "The hepatitis C virus encodes a serine protease involved in processing of the putative nonstructural proteins from the viral polyprotein precursor," Biochem. Biophys. Res. Commun., vol. 192, pp. 399-406, 1993.

[8] M. Hijikata, H. Mizushima, Y. Tanji, Y. Komoda, Y. Hirowatari, T. Akagi, N. Kato, K. Kimura, and K. Shimotohno, "Proteolytic processing and membrane association of putative nonstructural proteins of hepatitis C virus," Proc. Nat. Acad. Sci. USA, vol. 90, pp. 10773-10777, 1993.

[9] Y. Komoda, M. Hijikata, Y. Tanji, Y. Hirowatari, H. Mizushima, K. Kimura, and K. Shimotohno, "Processing of hepatitis C viral polyprotein in Escherichia coli," Gene, vol. 145, pp. 221-226, 1994.

[10] L. Tomei, C. Failla, E. Santolini, R. De Francesco, and N. La Monica, "NS3 is a serine protease required for processing of hepatitis $\mathrm{C}$ virus polyprotein," J. Virol., vol. 7, pp. 17-4026, 1993.

[11] T. Chambers, R. Weir, A. Grakoui, D. McCourt, F. Bazan, R. Fletterick, and C. Rice, "Evidence that the N-terminal domain of nonstructural protein NS3 from yellow fever virus is a serine proteinse responsible for site-specific cleavages in the viral polyprotein," Proc. Nat. Acad. Sci. USA, vol. 87, pp. 8898-8902, 1990.

[12] C. Steinkühler, A. Urbani, L. Tomei, G. Bisdiol, M. Sardana, E. Bianchi, A. Pessi, and R. De Francesco, "Activity of purified hepatitis C virus protease NS3 on peptide substrates," J. Virol., vol. 70, pp. 6694-6700, 1996.

[13] A. Urbani, E. Bianchi, F. Narje, A. Tramontano, R. De Francesco, C. Steinkühler, and A. Pessi, "Substrate Specificity of the hepatitis C virus serine protease NS3," J. Biological Chem., vol. 272, pp. 9204-9209, 1997.

[14] R. Zhang, J. Durkin, W. Windsor, C. McNemar, L. Ramanathan, and H. Le, "Probing the substrate specificity of hepatitis C virus NS3 serine protease by using synthetic peptides," J. Virol., vol. 71, pp. 6208-6213, 1997.

[15] I. Schechter and A. Berger, "On the size of active sites in proteases. I. Papain.," Biochem. Biophs. Res. Commun., vol. 27, pp. 157-162, 1967.

[16] Y. D. Cai and K. C. Chou, "Artificial neural network model for predicting HIV protease cleavage sites in protein," Adv. Eng. Software, vol. 29, pp. 119-128, 1998.

[17] A. Narayanan, X. Wu, and Z. R. Yang, "Mining viral protease data to extract cleavage knowledge," Bioinformatics, vol. 18, pp. s5-s13, 2002.

[18] N. Qian and T. Sejnowski, "Predicting the secondary structure of globular proteins using neural network models," J. Mol. Biol., vol. 202, pp. 865-884, 1988.

[19] R. Thomson, T. Hodgman, Z. R. Yang, and A. Doyle, "Characterising proteolytic cleavage site activity using bio-basis function neural networks," Bioinformatics, vol. 19, pp. 1741-1747, 2003.

[20] M. O. Dayhoff, R. Schwartz, and B. Orcutt, M. O. Dayhoff, Ed., "A model of evolutionary change in proteins: Matrices for detecting distant relationships," Atlas Protein Sequence Structure, vol. 5, pp. 345-358, 1978, Nat. Biomedical Res. Found., Washington, DC.

[21] M. Johnson and J. Overington, "A structural basis for sequence comparisons-An evaluation of scoring methodologies," J. Mol. Biol., vol. 233, pp. 716-738, 1993.

[22] S. F. Altschul, W. Gish, W. Miller, E. Myers, and D. J. Lipman, "Basic local alignment search tool," J. Mol. Biol., vol. 215, pp. 403-410, 1990.

[23] R. O. Duda and P. E. Hart, Pattern Classification and Scene Analysis. New York: Wiley, 2002.

[24] K. V. Mardia, J. T. Kent, and J. M. Bibby, Multivariate Analysis. London, U.K.: Academic, 1979.

[25] Y. Sun, T. Butler, A. Shafarenko, R. Adams, M. Loomes, and N. Davey, "Word segmentation of handwritten text using supervised classification techniques," Appl. Soft Computing, (in press).

[26] K. Torkkola, "Feature extraction by non-parametric mutual information maximization," J. Mach. Learning Res., vol. 3, pp. 1415-1438, 2003.

[27] V. Venkatraman, A. R. Dalby, and Z. R. Yang, "Evaluation of mutual information and genetic programming for feature selection in QSAR," J. Chem. Inf. Comput. Sci., vol. 44, pp. 1686-1692, 2004.
[28] C. Metz, "Basic principles of ROC analysis," Sem. Nucl. Med., vol. 8, pp. 283-298, 1978.

[29] R. L. Wilson and R. Sharda, "Bankruptcy prediction using neural networks," Decision Support Syst., vol. 11, pp. 545-557, 1994.

\section{Underdetermined Blind Source Separation of Temporomandibular Joint Sounds}

\author{
Clive Cheong Took, Saeid Sanei, Jonathon Chambers, and \\ Stephen Dunne
}

\begin{abstract}
The underdetermined blind source separation problem using a filtering approach is addressed. An extension of the FastICA algorithm is devised which exploits the disparity in the kurtoses of the underlying sources to estimate the mixing matrix and thereafter achieves source recovery by employing the $\ell 1$-norm algorithm. Besides, we demonstrate how promising FastICA can be to extract the sources. Furthermore, we illustrate how this scenario is particularly appropriate for the separation of temporomandibular joint (TMJ) sounds.
\end{abstract}

Index Terms-Filtering, nongaussianity, negentropy, sparsity.

\section{INTRODUCTION}

Blind source separation (BSS) is the problem of estimating source signals from their mixtures, without explicit a priori knowledge of the medium and the source signals. If the number of mixtures is less than the number of sources, the problem is termed underdetermined BSS (UBSS). It comprises of two stages: 1) blind identification of the mixing matrix $\mathbf{A}$ (of size $m \times n$ ); 2) source extraction. The generative model is given as

$$
\mathbf{x}(t)=\mathbf{A} \mathbf{s}(t)+\mathbf{v}(t)
$$

where $\mathbf{x}(t)$ represents the mixture signals detected by $m$ sensors at discrete time instant $t$, i.e., $\mathbf{x}(t)=\left[x_{1}(t), x_{2}(t), \ldots, x_{m}(t)\right]^{T} \in \Re^{m}$, and the source vector $\mathbf{s}(t)=\left[s_{1}(t), s_{2}(t), \ldots, s_{n}(t)\right]^{T} \in \Re^{n}$ where $^{T}$ denotes the transpose operation, and $\mathbf{v}(t)$ represents the additive noise vector. However in this work, we have modelled $\mathbf{v}(t)$ as an additional rank-1 source [1, pp. 221] assumed to be from other internal/external sources. Moreover, we employ both sparse and independent component analysis (SCA and ICA). ICA is a tool for BSS with the assumption that the sources are independent and unmixing is achieved by $\mathbf{y}(t)=\mathbf{W} \mathbf{x}(t)$, where $\mathbf{W}$ is the so called separating matrix (of size $n \times m)$ and $\mathbf{y}(t)$ is a vector of dimension $n$ containing the independent components (ICs) at discrete time instant $t$. On the other hand, SCA estimates the sparsest solution $\mathbf{s}(t)$ pertaining to (1) [2], where sparsity can be described as

$$
\left\{s_{i}(t) ; i=1, \ldots, n\right\}
$$

Manuscript received September 28, 2005; revised March 10, 2006. Asterisk indicates corresponding author.

${ }^{*}$ C. C. Took is with School of Engineering, Cardiff University, Cardiff CF24 3AA, U.K. (e-mail: cheongc@cf.ac.uk).

S. Sanei and J. Chambers are with School of Engineering, Cardiff University, Cardiff CF24 3AA, U.K. (e-mail: saneis@cf.ac.uk; chambers, @kcl.ac.uk).

S. Dunne is with GKT Dental Institute, Denmark Hill Campus, London SE5 9RW, U.K. (e-mail: stephen.dunne@kcl.ac.uk).

Digital Object Identifier 10.1109/TBME.2006.881789 

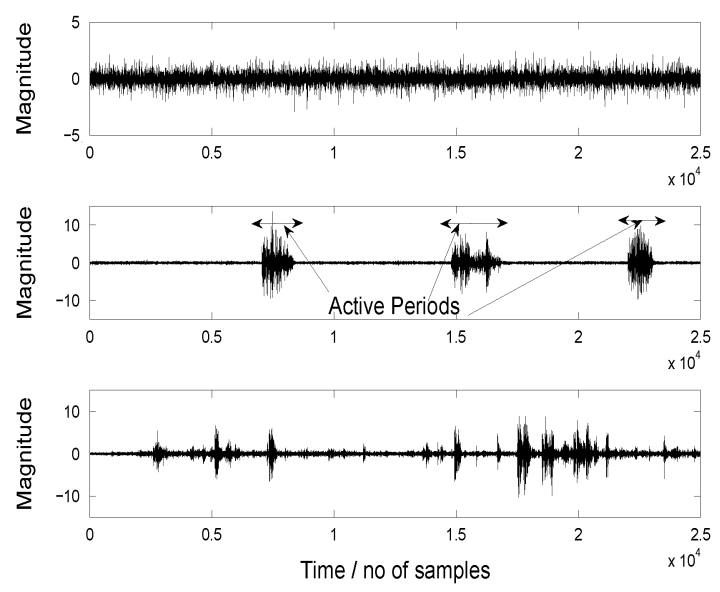

Fig. 1. The three source signals namely: the super-Gaussian noise, the click, and the crepitus. The SNR ratio is $8 \mathrm{~dB}$. Note the sparsity of the click (middle plot) where the click occurs in the three excitation regions. The same observation can be made for the crepitus signal (last plot).

where

$$
\begin{gathered}
\left|s_{k}(t)\right| \gg\left|s_{j}(t)\right| \quad \text { for } k \in 1, \ldots, n \\
\quad \text { and } \\
s_{j}(t) \approx 0 \quad \forall j \neq k
\end{gathered}
$$

where $s_{k}(t)$ is the $k$ th source signal. We address the problem of underdetermined BSS, where $\mathbf{A}$ is a $2 \times 3$ matrix as it pertains to the problem of separating TMJ sounds from two noisy observations. But, the algorithm presented herein can extend to the situation where $m=n-1$ whose results have been omitted due to limited space. The following hypotheses are assumed.

H1) All the source signals are super-Gaussian and mutually statistically independent.

H2) The columns of $\mathbf{A}$ are pairwise linearly independent.

H3) $\operatorname{kurt}\left(s_{2}(t)\right), \operatorname{kurt}\left(s_{3}(t)\right) \gg \operatorname{kurt}\left(s_{1}(t)\right)$ where $s_{i}(t)$ is the $i$ th source and $\operatorname{kurt}(\cdot)$ denotes the kurtosis.

H4) The two highly super-Gaussian source signals are sparse.

H5) The third source signal (with low kurtosis) has a substantially lower variance as compared to the other two sources, i.e., $\operatorname{var}\left(s_{2}(t)\right), \operatorname{var}\left(s_{3}(t)\right) \gg \operatorname{var}\left(s_{1}(t)\right)$ where $\operatorname{var}(\cdot)$ denotes the variance.

These hypotheses are valid for the problem in hand. The organization of the paper is as follows; the next section gives an overview of the temporomandibular disorder (TMD) problem. Section III details our algorithm. Section IV compares the performance of our algorithm against two algorithms, namely k-means clustering [3] (which relies on minimization of the distance between the data points and the assigned clusters) and the algorithm of Li et al. [4] (that scans for the sparse regions of the signals and clusters these regions to estimate the mixing matrix). Lastly, we suggest that FastICA can be a promising approach to extract such sources, and present our conclusions in Section V.

\section{THE TEMPOROMANDIBULAR DISORDER PROBLEM}

TMD is a collective term of medical problems related to the region of the mandible (lower jaw) and the temporal bone (skull) [5]. Two well-known TMJ sounds are: click and crepitus. Generally, the click is associated with the perforation of the disc holding the mandible and the temporal bone. Likewise, the crepitus hints at the presence of a degenerative joint disease (e.g., osteoarthrosis). A dental specialist has to distinguish between the TMJ sounds such as click, crepitus, and the background noise/interferences. However, the inherently subjective classification of these TMJ sounds makes it hard for the clinicians to determine the right pathology, leading to controversy [5]-[7]. Thus, it is particularly difficult for the dental specialist to diagnose TMD, when click, crepitus and background noise are all present within the TMJ sounds. For more information on TMDs, refer to [5]. Only two mixture signals can be recorded on a pair of stethoscopes placed in the auditory canals of the patient, while three source signals prevail in the mixture signals. Hence, a $2 \times 3$ UBSS scenario portrays itself in the source separation of TMJ sounds. In Fig. 1, the click is active for short and distinct periods, while the crepitus is a more noise-like signal, with approximately continuous active periods.

\section{DEVELOPMENT OF THE Algorithm}

1) First, we seek to estimate the two columns of the mixing matrix A corresponding to the two highly super-Gaussian sources by employing FastICA [8]. This is achieved by maximizing the negentropy $(\operatorname{Neg}(\cdot))$ of the pair of mixtures

$$
y_{1}(t)=\arg \sup _{w_{1}, w_{2}}\left(\operatorname{Neg}\left(w_{1} x_{1}(t)+w_{2} x_{2}(t)\right)\right)
$$

where $\mathbf{w}=\left[w_{1}, w_{2}\right]$, which is one of the rows of the separating matrix $\mathbf{W}$. The second row of $\mathbf{W}$ is estimated by (3), but followed by a deflationary orthogonalization. The key observation is that in UBSS FastICA will focus on the high kurtosis sources (H3). The two columns of $\mathrm{H}$ are estimated by inverting $\mathbf{W}$. The two TMJ sources can be estimated by $\mathbf{y}(t)=\mathbf{W} \mathbf{x}(t)$. Prior to using FastICA, preprocessing the mixtures with median filtering with a small window of three samples to yield $\mathbf{f}(t)=\left[\mathbf{f}_{1}(t) \mathbf{f}_{2}(t)\right]^{T}$ assists in attenuating the effects of the outliers to which FastICA is sensitive.

2) Next, our task is to estimate the third column corresponding to the noise source. We temporally mean filter $\mathrm{f}_{i}(t)$ with a window size greater than twice the duration of the maximum period $P_{\max }$ during which the two highly super-Gaussian sources are active. Fig. 1 shows these active periods. Although the window size is not known a priori, a large window can be used (H4). The mean filtering mitigates the two highly super-Gaussian source signals in both mixtures. Mean filtering of the signal $\mathrm{f}_{i}(t) i=1,2$ to yield $\mathrm{g}_{i}(t)$ is achieved as follows:

$$
\mathrm{g}_{i}(t)=\frac{1}{\mathrm{M}} \sum_{k=t-\mathrm{M}+1}^{t} \mathrm{f}_{i}(k) \quad i=1,2
$$

where $\mathrm{M}$ denotes the window size of the filter. Consider the periods when the two highly super-Gaussian source signals are active. We note the predominance of these two source signals in the mixture signals. Similarly, the converse is true when these highly super-Gaussian sources are not active. Hence, the averaging operation widens the active periods of the two highly super-Gaussian sources (in the mixture signals), while suppressing their amplitudes. Note the absence of high amplitudes (with respect to the TMJ sources) in the noise signal, resulting in a lower effect of the mean filtering on the noise signal. Now, the noise source predominates in the mixture signals.

3) Apply FastICA to estimate the third column of $\mathbf{A}$ pertaining to the weakly super-Gaussian source signal. However, FastICA may 
fail to estimate the column of $\mathbf{A}$ corresponding to the weakly super-Gaussian source because of its equivalence to projection pursuit (PP) [8]. Thus, we estimate two independent components (ICs) instead of one. The column corresponding to the IC with the minimum kurtosis is selected on the basis of $\mathrm{H} 4$ to form the last column of the estimated $\mathbf{A}$, i.e., $\hat{\mathbf{A}}$.

4) Apply the $\ell 1$-norm algorithm [2] to extract the source signals by minimizing the following cost function:

$$
\min \sum_{i=1}^{n}\left|s_{i}\right| \text { s.t. } \hat{\mathbf{A}} \mathbf{s}=\mathbf{x} \text {. }
$$

5) Alternatively, we can employ FastICA to perform source extraction. Begin by removing the mean-filtering (6) of the IC with the mininum kurtosis (from Step 3) to obtain the estimate of the least super-Gaussian source. However, there is still significant contribution of the most super-Gaussian source as seen in the first noise estimate in Fig. 3

$$
\mathrm{f}_{i}(t)=\mathrm{Mg}_{i}(t)-\sum_{k=t-\mathrm{M}+1}^{t-1} \mathrm{f}_{i}(k) \quad i=1,2 .
$$

Note that (6) does the opposite of (4), with the same notations for the purpose of clarity. To strengthen the presence of the source with the maximum kurtosis as the independent component, we can add any two distinct scaled version of the source with the maximum kurtosis. Applying FastICA to the two new mixtures improves the estimate of the weakly super-Gaussian source. See the first two plots of Fig. 3. This step is optional if one desires to fully solve the UBSS. However, the prime objective of our study is to extract the TMJ sources. The estimates of the highly nonGaussian sources are estimated from Step 1.

\section{EXPERIMENTAL RESULTS}

We have considered the case where TMJ sounds are the mixtures of click, crepitus, and a super-Gaussian noise. The click and crepitus were recorded with special stethoscopes connected to microphones placed at the openings of the auditory canals sampled at $8 \mathrm{KHz}$. Fig. 1 shows the sources: the weakly super-Gaussian noise, click and crepitus from top to bottom. The kurtoses of the noise, click, and crepitus (measured when each source exists separately) are, respectively, 3.0, 23.7, and 14.4. We model the noise with Laplacian distribution $P(s)=$ $(1) /(2 \lambda) \exp \{-(|s-\theta|) /(\lambda)\}$ where the variance $\sigma^{2}=2 \lambda^{2}$ and the mean $\mu=\theta$. The performance measure (PM) [9] which provides an indication of the difference between $\mathbf{A}$ and $\hat{\mathbf{A}}$ is employed here. However, PM requires both $\mathbf{A}$ and $\hat{\mathbf{A}}$ to have unit norm columns. $0 \leq \mathrm{PM} \leq 1$. PM equals to 0 if $\hat{\mathbf{A}}=\mathbf{A P}$ where $\mathbf{P}$ is a permutation matrix

$$
\begin{aligned}
\operatorname{PM}(\mathbf{A}, \hat{\mathbf{A}})=1-\left(\frac{1}{2 n} \sum_{i=1}^{n} \sup _{j}\left|\mathbf{A}^{T} \hat{\mathbf{A}}\right|_{i j}\right. & \\
& \left.+\frac{1}{2 n} \sum_{j=1}^{n} \sup _{i}\left|\mathbf{A}^{T} \hat{\mathbf{A}}\right|_{i j}\right) .
\end{aligned}
$$

The A matrix (generated randomly from a standardized normal distribution) used in this paper is

$$
\left(\begin{array}{ccc}
0.8999 & -0.9158 & 0.5984 \\
-0.4360 & 0.4017 & -0.8012
\end{array}\right)
$$

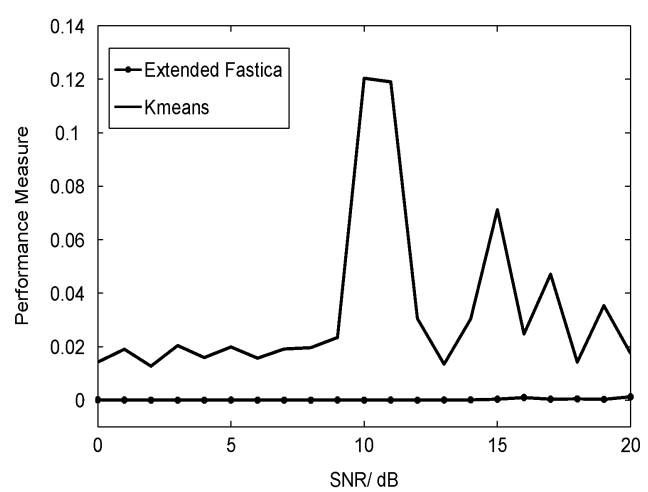

Fig. 2. The PM of our extended FastICA scheme plotted in "-*” and that of the $\mathrm{k}$-means clustering in "-" against SNR in decibels. The algorithm of Li failed to estimate the mixing matrix A.
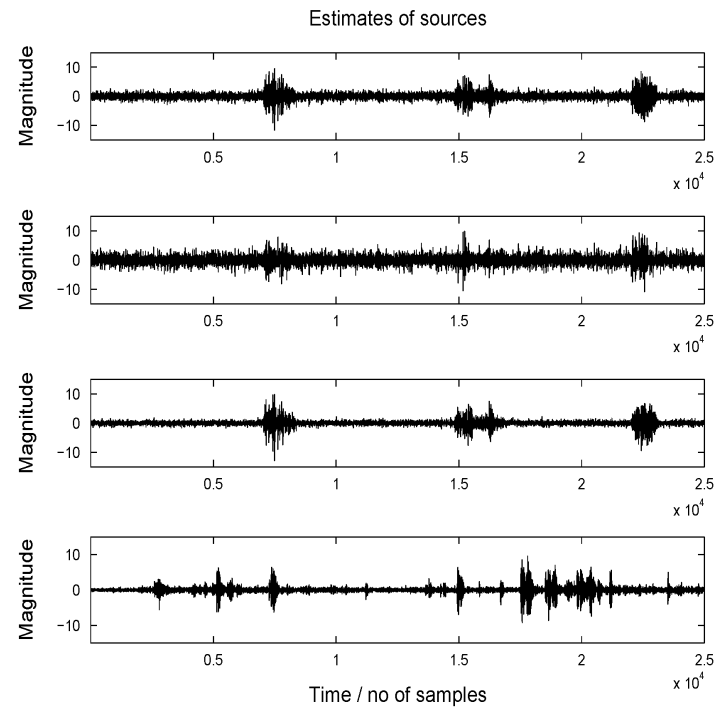

Fig. 3. The estimates of the three source signals. From top to bottom: Estimate of noise of Step 4, final estimate of noise, click and crepitus. The SNR ratio is 6 $\mathrm{dB}$. Note the significant presence of the click (most super-Gaussian source) in the first estimate of the noise.

The performance of our extended FastICA algorithm was measured against the k-means clustering [3] and the algorithm of Li et al. [4] as the signal-to-noise ratio (SNR) was varied. This is shown in Fig. 2. Thereafter, we visually demonstrate the potential of Step 6 of the extended FastICA algorithm to extract the sources in Fig. 3.

Comments: The maximum PM of the k-means algorithm is less than 0.14 which demonstrates its reasonable performance (which fluctuates due to its convergence depending upon initialization). On the other hand, our algorithm outperforms k-means (indicated by its much lower PM). The Li algorithm [4] failed to estimate $\mathbf{A}$, probably because the super-Gaussian noise source is always active. One of the assumptions of Li et al. is sparsity with respect to all the three signals.

\section{CONCLUSION}

This paper has shown how filtering can assist in solving the underdetermined blind source separation in the context of TMJ sounds. The common approach is geometrical such as the k-means and the algorithm of Li [4] exploiting sparsity, while our algorithm takes advantage of both the sparsity and the statistical properties of the source signals. In that respect, our algorithm is more efficient in its solutions than the k-means and Li's algorithms. The main idea of this paper is that provided we apply a linear transform to the mixture signals to suppress 
certain source signals within the mixtures, we can accentuate the prevalence of others. However, the challenge remains in finding more optimum linear transforms that perform source signals attenuation within the mixture signals.

\section{REFERENCES}

[1] A. Cichocki and S. Amari, Adaptive Blind Signal and Image Processing-Learning Algorithms and Applications. New York: Wiley, 2005.

[2] Y. Li, A. Cichocki, and S. Amari, "Analysis of sparse representation and blind source separation," Neural Computation, vol. 16, pp. 1193-1234, 2004.

[3] S. Roberts and R. Everson, Independent Component Analysis. Cambridge, MA: Cambridge Univ. Press, 2001.
[4] Y. Li, S. Amari, A. Cichocki, and D. W. C. Ho, "Underdetermined blind source separation based on sparse representation," IEEE Trans. Signal Process., vol. 54, no. 2, pp. 423-437, Feb. 2006.

[5] R. J. M. Gray, S. J. Davies, and A. A. Quayle, Temporomandibular Disorders: A Clinical Approach, 1st ed. London, U.K.: British Dental Assoc., 1995.

[6] C. S. Greene, N. D. Mcneill, C. Clark, and G. T. Truelove, "Temporomandibular disorders and science: A response to the critics," J. Prosthetic Dentistry, vol. 80, p. 214, 1998.

[7] G. A. Toolson and C. Sadowsky, "An evaluation of the relationship between temporomandibular joint sounds and mandibular movements," J. Craniomandibular Disorders: Facial Oral Pain, vol. 5, p. 187, 1991.

[8] A. Hyvärinen, J. Karhunen, and E. Oja, Independent Component Analysis. New York: Wiley, 2001.

[9] F. C. Meinecke, S. Harmeling, and K. R. Muller, "Robust ICA for super-gaussian sources," in Proc. 5th Int. Conf. Independent Component Analysis and Blind Source Separation, 2004, vol. 5, pp. 217-224. 